\title{
Après le tournant iconique
}

\section{Tania Vladova}

\section{OpenEdition}

\section{Journals}

Édition électronique

URL : http://journals.openedition.org/imagesrevues/3996

DOI : 10.4000/imagesrevues.3996

ISSN : 1778-3801

\section{Éditeur :}

Centre d'Histoire et Théorie des Arts, Groupe d'Anthropologie Historique de l'Occident Médiéval, Laboratoire d'Anthropologie Sociale, UMR 8210 Anthropologie et Histoire des Mondes Antiques

\section{Référence électronique}

Tania Vladova, « Après le tournant iconique », Images Re-vues [En ligne], Hors-série 5 | 2016, mis en ligne le 18 décembre 2016, consulté le 30 janvier 2021. URL : http://journals.openedition.org/ imagesrevues/3996 ; DOI : https://doi.org/10.4000/imagesrevues.3996

Ce document a été généré automatiquement le 30 janvier 2021.

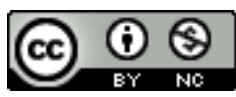

Images Re-vues est mise à disposition selon les termes de la Licence Creative Commons Attribution -

Pas d'Utilisation Commerciale 4.0 International. 


\section{Après le tournant iconique}

Tania Vladova

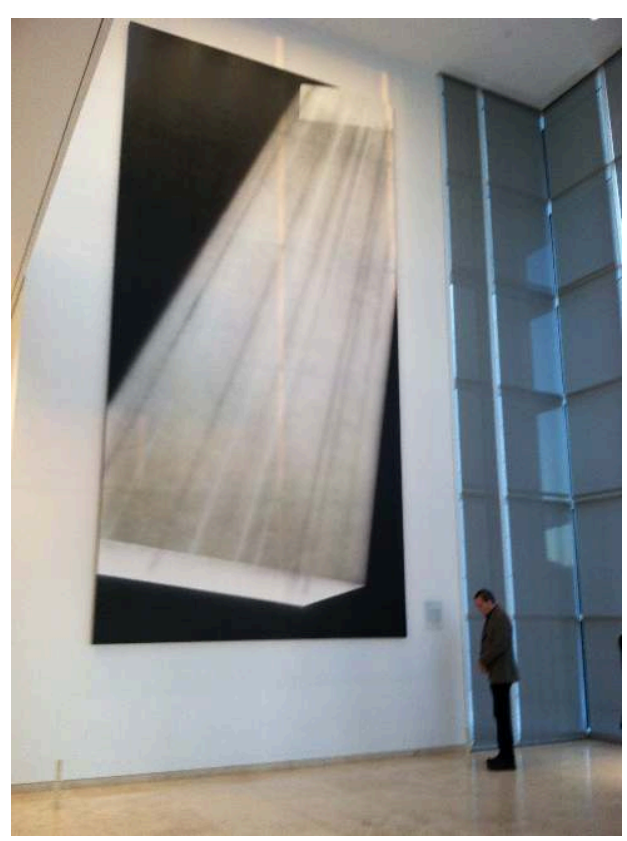




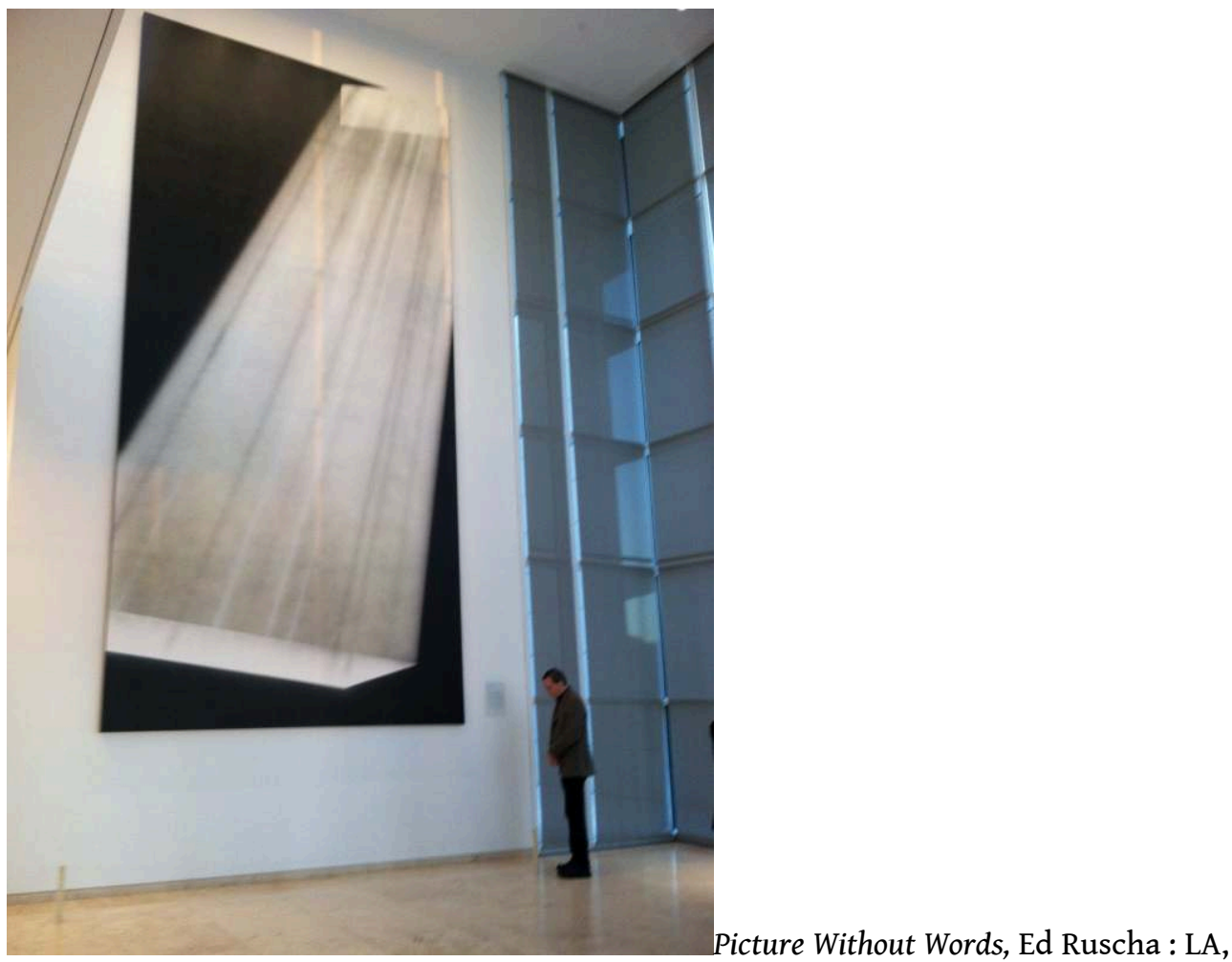

Getty, 1997

$\mathrm{Au}$ centre de ce volume intitulé Après le tournant iconique demeure l'intuition, plutôt que le constat, d'un affaiblissement sinon d'une sortie ou d'une fin du tournant iconique. Tout en gardant à l'esprit les acquis de ce tournant, cela oblige à repenser les rapports entre images et textes en réévaluant l'héritage iconologique et structuraliste. Si sortie il y a, elle n'est pas liée à un quelconque affaiblissement du rôle des images, mais plutôt à la nécessité de changer de perspective, de repenser à nouveaux frais notre rapport à l'art et à la matérialité de ses œuvres, mais aussi de passer outre l'opposition stérile et insoluble entre images et textes qui, dans le manège entre tournant linguistique et tournant iconique, a traversé tout le XXe siècle. Cette sortie ou postérité serait donc liée à une prise de distance libératrice en ce qu'elle permettrait d'adopter une vue synoptique seule capable d'embrasser la complexité du réel.

Ernst Cassirer, dans son essai " Langage et art $»^{1}$, écrivait que l'art (et a fortiori l'image) et le langage sont deux points focaux sur le monde. Plus encore, ils apparaissent comme deux attitudes, deux entrées qui, pour complémentaires qu'elles soient, constituent deux manifestations distinctes de la pensée par le biais desquels le monde se donne à percevoir différemment. À l'encontre de tout essentialisme, Cassirer y voyait des fonctions et des énergies humaines autonomes et originales. C'est précisément grâce à ces énergies que nous parvenons à construire et à organiser le monde de nos perceptions, de nos concepts et de nos intuitions. De ce fait, art et langage sont affirmés dans leur valeur non seulement reproductive, mais productive et constructive, c'est-àdire cognitive. Mettant en avant les processus de symbolisation, et insistant sur l'intensification qui dans le cas de l'art vient s'ajouter aux fonctions d'expression, représentation et signification pure propres au langage, Ernst Cassirer ouvrait ainsi une des voies possibles qui permettent de dépasser la dualité maîtresse entre approches 
formaliste et iconologique, et fondait la possibilité de considérer les œuvres d'art, images et textes à partir des différentes fonctionnements, interférences mais aussi rituels dans lesquels ils sont pris.

Cependant, tout au long du XXe siècle, la pensée des images a été nourrie par l'héritage linguistique (Saussure) et iconologique (Panofsky), avec leurs déclinaisons sémiotique et structuraliste. La révélation psychanalytique (de Freud à Lacan) a, quant à elle, malgré son intérêt pour les processus de symbolisation, contribué à l'affirmation de la parole, du verbe, comme logos. Dans la bonne tradition exégétique, ces orientations de la pensée perpétuaient la lecture des «images ». Voir et montrer se retrouvaient pris, avant même d'être pleinement réalisés, dans une logique et dans des structures propositionnelles, dans l'ordre formel de la langue. L'efficacité de la critique apportée par Richard Rorty dans L'homme spéculaire ${ }^{2}$ d'une tradition philosophique avant tout visuelle, spéculative parce que spéculaire, spectatrice de la connaissance car nourrie de métaphores, a marqué le siècle. Comme le montre clairement Bernd Stiegler dans l'introduction au volume Iconic Turn qui donnait le coup d'envoi de la revue francoallemande Trivium en 2008 :

Un paradigme langagier est devenu constitutif de domaines aussi divers que la philosophie analytique, la sémiotique et la sémiologie, le structuralisme et le poststructuralisme (ou néo-structuralisme), ou encore l'herméneutique. « Le monde comme texte » a été érigé en une nouvelle métaphore, qui n'a pas tardé à devenir aussi dominante que la métaphore spéculaire de la tradition philosophique critiquée par Rorty ${ }^{3}$.

2 Cette ambigüité constitutive a nourri plus d'une génération d'historiens et théoriciens de l'art français au XXe s. ayant exploré autant de territoires où s'évertue l'image entre l'imaginaire, le pouvoir de la représentation ou l'iconographie analytique, dans un effort plus ou moins marqué de prise de distance avec l'iconologie de Panofsky. De Pierre Francastel à Daniel Arasse, d'Émile Benveniste à Louis Marin et de Maurice Merleau-Ponty à Hubert Damisch, sans oublier Roland Barthes, Jean-François Lyotard ou Jacques Derrida, ou encore Jacques le Goff, ce qui a été désigné outre-Atlantique par un nom national, symptomatique de la largesse et de la porosité des propositions French Theory, a frayé son chemin aux croisements des traditions linguistiquesiconologiques et phénoménologiques-psychanalytiques, faisant sienne cette ambigüité constitutive du tournant linguistique. La pensée de l'image se construisait donc, à travers les multiples perspectives ouvertes par l'«école» française, sur fond de logique propositionnelle et d'imaginaire métaphorique.

Cela permet peut-être de comprendre la difficulté à constituer une école française homogène analogue aux Visual Studies et à la Bildwissenschaft, et le temps des réajustements qu'a nécessité en terrain français le tournant iconique. Affirmé à partir du tout début des années 1980, lorsqu'il fut nommé par W.J.T. Mitchell à Chicago par la formulation " pictorial turn », et par Gottfried Boehm à Bâle comme « iconic turn »", il signait un infléchissement de la pensée dans le sens de l'image, suite au constat d'une faiblesse théorique. La force de ce changement de perspective qui a trouvé un terrain fertile dans l'ensemble des sciences humaines et sociales, était évidente. Elle s'enracinait dans le sentiment d'une urgence à rendre compte de l'impact social et cognitif des images, dans la nécessité d'un changement de la vis théorique même ${ }^{5}$. Il n'était plus du tout question d'aller dans le sens de la pensée spéculaire et métaphorique dénoncée par Rorty, mais de reconnaitre l'impact culturel et l'irréductibilité fondamentale de l'image au logos discursif. Ce tournant a focalisé, de 
façon souvent impérieuse, les regards vers les valeurs cognitives, gnoséologiques, scientifiques, historiques, anthropologiques, sociologiques, propres aux images seules. Le tournant est un terme qui implique l'infléchissement de la perspective. Formulé d'après le paradigme de Thomas Kuhn, l'épistémè de Michel Foucault et sans doute tributaire de la rhétorique des styles -, il insiste sur le processus de changement, sur la transformation, sur la prise d'une nouvelle direction, plutôt que sur le socle stable. De cette façon, ce qui est cerné par l'idiome tournant pictural ou iconique n'est pas tant le constat d'une multiplication exponentielle de la quantité et la généralisation des moyens de production d'images, mais plutôt la prise en compte d'un changement, d'un état d'instabilité, d'un moment charnière qui modifie les enjeux, et oblige à reconsidérer les outils qui nous donnent accès à notre culture ${ }^{6}$.

$\mathrm{Au}$ cœur même de ce moment charnière et dans une sorte de radicalisation du tournant linguistique plutôt que par opposition à celui-ci, Gottfried Boehm s'est proposé de comprendre l'image comme «logos", comme processus qui engendre du sens, qui a une fonction "aussi primitive que celle du langage ». Ainsi, à partir d'une tradition allemande philosophique et herméneutique, grâce entre autres à Ernst Cassirer (deixis), Husserl (Abbild) et Wittgenstein (voir comme et voir dans), Boehm se demandait comment ce logos visuel, iconique, non verbal produit une pensée autonome ${ }^{7}$. Le constat fondamental que l'homme éprouve un désir de reconnaître et comprendre les images, rendait nécessaire cette tentative de dégager une logique qui leur est propre et inaliénable, une logique dont on pourrait retracer les opérations minimales (pouvoir déictique, faculté de procéder par contrastes, production de différences). Le tournant vers l'iconique consistait alors à reconnaitre le fait que les images engendrent du sens grâce à une logique non-prédicative, puisqu'elles ne sont ni écrites ni lues, mais perçues. Que des images jusque-là inconnues puissent apporter des savoirs que nous sommes incapables d'acquérir autrement fonctionnait comme preuve pour Boehm tant de leur exclusivité que de leur puissance cognitive. Grâce à ces conclusions, le fondateur du pôle Eikones. Critique de l'image à Bâle, s'employait alors à montrer comment une image fait sens et touche aux fondements de notre culture. Cette approche, empreinte de la tradition philosophique et herméneutique européenne, se différenciait tant de l'ambition de l'iconologie immanquablement aboutissant à une lecture des images, que des Visual Studies, qui en revanche étudient avant tout les circulations et implications sociales et médiatiques de l'iconique. Le socle des réflexions de Boehm, la critique de l'image (Bildkritik) touche au sens kantien du terme, puisqu'il recherche, par delà la variété empirique, des conditions de possibilité et des opérations élémentaires de l'image. C'est à partir de ce socle qu'il explore l'hypothèse, naturaliste et anthropologique, selon laquelle la représentation colle aux objets, et se présente à la fois comme qualité de la matière et besoin de la perception (comme le montrent par exemple les ready-made) ${ }^{8}$.

Si Gottfried Boehm aspirait à une science de l'image, à partir de la tradition philosophique européenne et de la corporéité de la tradition picturale classique, autrement dit sans se pencher sur des images quotidiennes, marginales, médiatiques, William John Thomas Mitchell, chantre du tournant pictural et des Visual Studies, insistait au contraire sur l'immatérialité, sur les flux et circulations des images dans les médias où se joue constamment l'oscillation entre l'illusion de plus en plus parfaite de transcender tout support matériel et le fait d'apparaître toujours sur un support. C'est précisément là, dans cet écart énergétique que Mitchell situait dès les années 1980 l'impossible tâche des iconoclasmes, car la destruction de sa présence matérielle ne 
faisait qu'augmenter l'énergie de l'image, à commencer par le fait de se rendre compte du spectacle de la destruction lui-même ${ }^{9}$. L'énergie propre à l'image guide l'approche que l'on pourrait nommer socio-vitaliste de W.T.J. Mitchell mêlant vie, biologie, et désirs des images. Dans Que veulent les images ${ }^{10}$ il assimile ainsi le moment de reconnaissance d'une image à une rencontre intersubjective ce qui lui permet d'analyser les images par le biais de leurs volitions. Que ce soit le face à face entre deux personnes ou entre l'image et son observateur, dans les deux cas il en va dans la perspective de l'historien de l'art et de la littérature de l'Université de Chicago de la compréhension de "l'autre " au sens large, d'une altérité qui se donne à voir et que l'on cherche à comprendre, tout en reconnaissant à quel point les arts visuels sont pris dans des croyances populaires et autres enjeux idéologiques ${ }^{11}$. Cette perspective résulte entre autres de la volonté de W.J.T. Mitchell de libérer l'image du carcan disciplinaire traditionnel de l'histoire de l'art et de l'esthétique de façon à en faire non pas un modèle mais une « cible » (target) des sciences sociales.

La même volonté de dépassement de l'histoire de l'art et de l'esthétique comme territoires privilégiées de l'image se retrouve également, bien que dans une perspective et avec des ambitions nettement différentes, chez Hans Belting et Horst Bredekamp, deux autres acteurs importants dans l'institutionnalisation des études sur l'image en Allemagne (que l'on pense au Centre d'art et technologie des médias à Karlsruhe, fondé par Hans Belting, et au laboratoire interdisciplinaire Bild, Wissen, Gestaltung, à la Humboldt Universität où œuvre Bredekamp, ou encore au pôle Eikones à Bâle dont il a été question). Belting définit une approche anthropologique forte: non seulement elle sollicite des objets et rituels anthropologiques dans la compréhension des images, mais aussi, dans la lignée d'Aby Warburg, elle insiste sur le rôle crucial du corps humain comme réservoir singulier et pathique d'images. Comprises comme sources premières de la vie affective, psychique et intellectuelle de l'humanité, les images se retrouvent ainsi questionnées dans une perspective anthropologique fondamentale ${ }^{12}$.

La position de Horst Bredekamp, quant à elle, est bien plus tributaire de la Kunstwissenschaft, vaste entreprise interdisciplinaire du début du XX $\mathrm{XX}^{\mathrm{e}}$ siècle, dont une des grandes figures institutionnelles est le philosophe et psychologue Max Dessoir. Ce dernier proposait de placer l'art - «la plus libre des activités humaines » - au centre d'une réflexion interdisciplinaire ${ }^{13}$ et d'une nouvelle science composite, allgemeine Kunstwissenschaft, conçue à partir du clash initié au XIX ${ }^{\mathrm{e}}$ siècle entre métaphysique et empirisme, entre philosophe spéculative et positivisme scientifique, entre esthétique et théorie de l'art. L'accent ayant été déplacé de l'art vers l'image à la fin $\mathrm{du} \mathrm{XX}$ siècle, Horst Bredekamp élabore sa théorie des actes d'images (Bildakte) dans le cadre de la vaste mouvance de la Bildwissenschaft ou science des images. Optant pour une perspective pragmatiste, dans le sillage de Charles Sanders Pierce et John Austin, il interroge plus particulièrement la portée cognitive, sociale, politique et historique des images. Selon sa perspective, l'image n'est pas juste un contenu passif de la conscience. "L'image est un acte et non une chose. L'image est conscience de quelque chose", écrivait Sartre dans l'Imaginaire en $1940^{14}$. Bredekamp rebondit à partir de ce même propos : «l'image est acte », mais qu'il emprunte plutôt à la Critique de la vie quotidienne d'Henri Lefebvre ${ }^{15}$, mentionnant également Philippe Dubois qui évoque lui aussi des images-actes $^{16}$. Une troisième grande source dans laquelle puise Bredekamp est l'anthropologie, notamment à travers la théorie de l'agentivité des artefacts d'Alfred $\mathrm{Gell}^{17}$, et Le pouvoir des images de David Freedberg ${ }^{18}$. Dans cette perspective, les images sont envisagées comme étant à l'origine de pratiques, ayant le pouvoir d'instituer des 
formes d'histoire, des expériences, des actions, d'agir comme des armes selon le mot de Bredekamp, et même de constituer leur propre théorie, tel le rêve d'une théorie des images par les images.

Conçues tantôt dans leur corporéité anthropologique et faisant partie de nos corps mêmes (Hans Belting), tantôt comme configurations visuelles qui font sens par des opérations logiques qui leur sont propres (Gottfried Boehm), ou encore comme actes (Horst Bredekamp), ou bien comme des symptômes, des blessures, des objets désirants (T. W. J. Mitchell), les images se sont donc imposées des deux côtés de l'Atlantique comme objet privilégié, comme laboratoire de notre modernité selon la formulation de Boehm ${ }^{19}$.

Telle a été la frappe décisive des Visual Studies et de la Bildwissenschaft, une frappe qui a été présentée et interrogée dans des anthologies devenues désormais incontournables et qui questionnent les apports des différents centres de réflexion interdisciplinaire sur les images. Pour en citer quelques références, que l'on considère, Iconic Turn. Die Neue Macht der Bilder ${ }^{20}$, ou le premier numéro déjà cité de la revue Trivium "Iconic Turn » (éd. Bernd Stiegler), mais aussi les recueils de textes-clés Penser l'image ${ }^{21}$, ou encore Bildtheorien aus Frankreich. Ein Handbuch ${ }^{22}$, ainsi que Visual Theory: painting and interpretation ${ }^{23}$, ou Visual Culture: The Reader ${ }^{24}$. Outre ces éditions de textes-sources, mentionnons le travail initié par le Réseau thématique pluridisciplinaire Visual Studies (IRHIS-CNRS), un des principaux centres de recherches interdisciplinaires en culture visuelle en France créé en 2010.

L'affranchissement de l'image de l'emprise du langage, du texte, du verbe, a été un point d'honneur du tournant iconique. En comparaison, la question du rapport entre images et sons, la prise en compte de l'environnement sonore, bien qu'ayant connu une résurgence avec la reconnaissance d'un tournant acoustique ${ }^{25}$, et avec la conceptualisation des paysages et images sonores, peine encore à s'imposer comme facteur puissant et aussi incontournable dans les processus de constitution du sens, malgré certains travaux récents qui vont dans ce sens ${ }^{26}$.

Le " tout image » s'est donc constitué, sur le plan historique, par opposition tranchée contre le (ou en tant qu'approfondissement du) mot d'ordre structuraliste "tout texte ", l'un tout comme l'autre souffrant des mêmes raccourcis ${ }^{27}$. Cependant, le rôle fondateur de l'image pourrait à son tour être remis en question et on tomberait facilement dans le paradoxe de l'œuf et de la poule. À titre d'exemple, et pour n'en prendre qu'un seul, nulle part ailleurs les mots ne reviennent avec plus d'insistance que dans Picture without Words d'Ed Ruscha : LA, Getty, 1997.

Certes, l'image a une portée heuristique. Heuristique, car à la manière d'un outil optique elle permet d'aller au-delà de ce que l'œil humain peut voir tout seul, heuristique puisqu'elle a sans nul doute une part irréductible au langage ou à la musique et à toute autre forme d'expression, heuristique aussi car au lieu d'engager le spectateur dans des explications fastidieuses, elle montre tout d'un seul mouvement. Elle s'impose comme évidence, conséquence d'un mode opératoire dont elle seule peut nous fournir les clés de la perception et de la compréhension.

Si au moins depuis Sartre les images se montrent comme autant de processus plutôt que des objets, pris dans des jeux de forces ${ }^{28}$, présentent un intérêt bien plus large que l'histoire de l'art, l'esthétique ou la philosophie de l'art peuvent prétendre leur accorder, quel autre lieu que Images Re-vues se prêterait mieux à cette remise en question sinon du tournant iconique, du moins de sa durée. Cette revue, consacrée à l'histoire, l'anthropologie et la théorie de l'image, a été créée en 2005, en pleine 
effervescence française des querelles et autres débats autour des images. À l'époque, les affrontements sur la portée du visuel faisaient rage dans les milieux académiques, et en bonne cure d'interdisciplinarité, le rapprochement des scientifiques, historiens de l'art, philosophes, sociologues et autres anthropologues portait, infailliblement, un nom: l'image. Des diagrammes aux fétiches et aux églises, des études statistiques aux partitions musicales, aux écrans de télé et à l'imagerie médicale, des preuves archéologiques aux documentations de terrain des anthropologues et jusqu'à l'étude de la structure des cristaux et aux réseaux de neurones, tout passait par un dénominateur commun, annoncé de façon prémonitoire presque comme une révolution de la pensée : l'image. À cette époque, quelques enthousiastes ${ }^{29}$, doctorants et jeunes docteurs, décidaient d'offrir au public francophone une revue académique en ligne et en libre accès sur l'image, entreprise alors plutôt pionnière en France. Revue interdisciplinaire, revue sur l'image, toutes époques confondues et toutes approches autorisées, Images Revues a vu le jour dans un petit bureau de l'EHESS. On partait de zéro, tout était à inventer, et les défis ont été nombreux, allant de la difficulté liée à la légitimité scientifique d'une revue en ligne et en libre accès, aux nombreux soucis liées à une législation encore vacillante sur les droits des images, sans parler des aspects matériels. Depuis, on connaît l'histoire : les publications et revues scientifiques en ligne, en France comme à l'étranger, ont connu un véritable essor. Dès les premières années de sa création, la revue a trouvé sa place, et l'exigence mais aussi les questionnements sur l'image nous ont valu un intérêt assez conséquent. Légitimée par sa présence dans des bibliographies académiques à travers le monde, par son inclusion dans la liste des revues AERES et hébergée sur le portail revues.org, cette entreprise s'est affirmée car elle répondait sans doute non seulement à un phénomène de mode, mais à un questionnement à la fois très ouvert et très actuel, car lié à des transformations qui s'attaquaient à notre rapport même à la culture, à la connaissance et, last but not least, au langage.

C'est donc à partir du cœur de ce lieu privilégié accordé aux images, de cette tribune analytique et critique impulsée par le tournant iconique, qu'il convient aujourd'hui, dix ans après son instauration, et quitte à s'engager dans une franche contestation du pouvoir absolu et totalisant des images, de se demander : est-il encore, toujours, et de la même manière important de centrer les discussions sur l'image? N'est-ce pas aujourd'hui un terme galvaudé, une idée qui à force de fuser de partout a fini par perdre de sa puissance heuristique, si tant est que les débats initiés dans les années 1980 ont apporté de réelles nouveautés par rapport à la riche tradition philosophique, autre que la focalisation impertinente des regards, de tous les regards, sur ce qu'on voit, sur ce qui nous semble signifiant par le fait d'être vu et identifié, sur sa manière de fonctionner, sur ce enfin qui "nous regarde"? Sommes-nous dans une ère postimages? En quoi et comment ce tournant a-t-il infléchi les équilibres et repositionné les images dans le spectre de la connaissance? Autrement dit, ce tournant iconique, est-il encore et toujours opérant, actif, en vigueur? Ou bien, le tumulte passé et quelques leçons apprises, il est temps d'ôter la loupe braquée sur les supports et les surfaces et de revenir à la matérialité des œuvres, et donc à l'art?

Dans le volume présenté ici, l'image est prise dans une acception large, qui comprend aussi bien les configurations iconiques matérielles que les images littéraires et mentales alors que par texte, on entend ce qui ressort du registre du langage verbal (y compris la parole et le métatexte). Autrement dit, le couple image et texte reprend ici la distinction cassirerienne entre art et langage en tant que dualité formatrice pour 
toute notre culture. Cependant cette distinction n'entend pas essentialiser les deux pôles. Au contraire, au moins depuis les collages dada et futuristes, depuis l'art conceptuel et narratif, reconnaître que les images peuvent agir comme textes et que les textes peuvent fonctionner comme images devrait relever du sens commun. Pour le dire avec Bernard Vouilloux et en évitant la focalisation essentialiste, les rapports entre régimes iconique et discursif restent, et resteront sans doute, peu importe le terme qu'on mettrait en premier, au cœur des débats sur les processus dans lesquels nous nous engageons pour percevoir, exprimer et comprendre le monde. Ou bien, suivant Jacques Morizot $^{30}$, on pourrait prendre l'exemple des indiscernables pour pointer la question de l'intentionnalité comme une des voies susceptibles de passer outre les propriétés distinctes des images et des textes (dépiction et description), pour orienter la discussion sur les images et les textes vers les processus de simulation et l'expérience de la fiction. Ce faisant, il ne s'agissait évidemment pas de gommer les différences, l'abîme qui sépare ces deux modes distincts de constitution de sens et d'accès au monde, mais de rendre compte de possibles voies de sortie de la sémiotique. Une telle voie est proposée par vaste programme de naturalisation de l'esprit, par l'intérêt pour les processus virtuels engagés par les images, la simulation fictionnelle, et les mécanismes psycho-cognitifs qui diminuent sensiblement le rôle épistémologique que le langage jouait autrefois.

La perspective adoptée ici explore en revanche un cheminement différent, quitte à rejoindre par moments des arguments sémiotiques. Le volume Après le tournant iconique comprend les versions développées d'une sélection de contributions au colloque « Image et texte : retour au texte? ? qui se sont tenues au Centre de recherches sur les arts et le langage-EHESS en mai 2012. Ces journées proposaient une rencontre non seulement entre l'image et le texte, et respectivement l'histoire de l'art et l'esthétique, avec les études littéraires et linguistiques en tant qu'exemplaires des deux pôles de réflexion représentatives du Centre de recherches sur les arts et le langage (CRALEHESS/CNRS). Par delà ce cadre institutionnel, elles entendaient contribuer à la réflexion sur notre perception du monde à partir d'une remise en cause de l'hégémonie acquise par l'image aux dépens du texte. Autrement dit, la question qui se posait était : et s'il était temps d'assumer que l'autonomie supposée des images n'est qu'un leurre, que les images n'ont plus besoin d'être autant protégées de la domination des textes, du verbe, du langage, de l'écrit, de la littérature?

La visée de ces journées était non pas de revenir en arrière, vers une proposition qui se revendiquerait du modèle des word\&image studies impulsées, pour des raisons à la fois historiques et disciplinaires, dès les années 1960 par des études littéraires et qui demeurent pour cela fondées soit sur une priorité du texte soit sur l'assimilation. Bien au contraire, notre proposition était de reprendre la question à partir du point où nous nous trouvons: l'après tournant iconique. La place massive accordée à l'image aujourd'hui est symptomatique d'un déplacement de perspective dans la considération de notre culture. C'est précisément cette bascule qu'affiche le titre du présent volume : l'image devant le texte affirme le déplacement de perspective, ainsi que la volonté de se démarquer d'un cadre disciplinaire, volens ou nolens, issu du structuralisme, filiation pas toujours ouverte ou consciente et qu'il est temps de réexaminer. Se situer dans la postérité du tournant iconique, forts de ses acquis, impliquait également la prise en compte d'une question cruciale : pour irréductibles qu'ils soient l'une à l'autre, image et texte restent inextricablement liés dans la formation même de la pensée, qu'elle soit artistique ou non. Par delà les revendications d'une autonomie iconique ou langagière 
intenable, les articles ici présentés proposent d'examiner en quoi la présence exacerbée du paradigme de l'image dans les arts et dans les sciences sociales lors des dernières décennies a eu comme contrecoup un réajustement des relations entre image et langage, un retour en force vers leurs modes d'être, d'agir et de concourir ensemble.

Les réflexions suivent deux axes majeurs. D'un côté elles interrogent la façon dont ces débats affectent les positionnements des disciplines concernées et leur histoire (esthétique, histoire de l'art, littérature, anthropologie, sciences cognitives, linguistique, sociologie: Bernard Vouilloux, Muriel Van Vliet, Filippo Fimiani). De l'autre, elles questionnent le statut même accordé aujourd'hui à l'image, au langage, au texte et à leurs interactions dans les créations de l'art contemporain (Jacques Leenhardt, Georges Roque, Marie-Aimée Lebreton).

Les contributions de littéraires, historiens de l'art, philosophes et anthropologues permettent de dresser ainsi un état des lieux de la relation image-langage dans les arts et dans les disciplines qui s'en occupent aujourd'hui. Après le tournant iconique, les multiples tentatives de capter une orientation avant tout iconique de notre actualité (le savoir, ses pratiques et ses transmissions passeraient désormais prioritairement par les images), nécessitent sans doute un retour à la question de la matérialité des œuvres. Une matérialité dont le langage ne saurait que faire partie intégrante.

À considérer le problème dans la perspective de l'histoire des idées, un des risques, entraîné par le tournant iconique, très clairement formulée par Muriel Van Vliet, est le "passage périlleux» d'une philosophie du sujet se revendiquant d'une "conception représentationnelle du monde », d'un « universalisme stérile aveugle à la spécificité du visuel », à " une déconstruction du sujet qui viderait l'individu de toute teneur et, en voulant faire droit à la toute-puissance des images 'fascinantes', ferait de son côté bon marché de toute préoccupation éthique véritable, perdant le sens même de la belle notion d'humanisme ouvert encore revendiquée par Lévi-Strauss [...] $\rrbracket^{31}$. C'est à partir de cette brèche que Muriel Van Vliet analyse finement le rapport entre art et langage chez Ernst Cassirer dans le contexte de deux tournants langagiers : le premier impulsé par Humboldt et pointant vers l'iconologie de Panofsky, et le suivant qui à travers la notion de forme (Gestalt), est pensé dans une continuité entre la morphologie goethéenne et le structuralisme de Claude-Lévi-Strauss.

Partant de l'exergue de Jean-Luc Godard, qui pointe plus qu'une simple complémentarité, un retour aux choses-même: "Mot et image, c'est comme chaise et table: si vous voulez vous mettre à table, vous avez besoin des deux.", Georges Roque dans son «Esquisse d'une rhétorique des énoncés verbo-iconiques » adopte une perspective plus sémiotique et cherche à cerner la voie du milieu: un équilibre dans les interactions, le travail de concert et les diverses formes de synergie entre images et textes dans les énoncés mixtes. Entre affiches et annonces, l'«impérialisme linguistique " du groupe $\mu$ est considéré sous l'angle de la rhétorique et la théorie littéraire, de Barthes à W.T.J. Mitchell.

La focalisation sur ce qu'on a dénommé «culture visuelle " peut être comprise en tant que réaction à un statu quo jugé décevant. La volonté tant de rendre compte des vastes implications socio-culturelles et anthropologiques des images, que le désir de débarrasser l'image de l'emprise du texte, ont eu comme conséquence une certaine mise au placard de l'intérêt pour le texte, voire une mise à mal des disciplines littéraires qui s'en sont accommodé en élargissant leur outillage disciplinaire, et en se tournant de plus en plus vers l'anthropologie, vers l'histoire de l'art ou vers les sciences cognitives, pour n'en citer que quelques unes, si ce n'est en s'éclipsant ${ }^{32}$. À rebours de 
cette tendance se déploie la fresque historique à la croisée des disciplines que propose Bernard Vouilloux avec l'article « La peinture dans la rumeur du langage ». La parole poétique (la poématique), tout comme les formes et couleurs, est passée au crible d'une exploration tour à tour philologique, poétique et philosophique qui, à l'encontre du logocentrisme contemporain en littérature ou d'un prosaïsme bavard convoque les possibilités du langage, la poïese. Par-delà la signification linguistique, la parole poétique figure la relève des choses et éveille jusqu'au silence.

La relève du silence, sous une autre forme, non moins éloignée de la stricte signification linguistique, est discutée dans l'article de Marie-Aimée Lebreton "Qu'estce qu'une image sonore?», qui, quant à elle, problématise le désarrimage entre musique et poésie pour se concentrer sur la nature duale des sons: acoustique et plastique, à partir de la voie ouverte par Duchamp et Cage, et jusqu'à l'architecture sonore de Xenakis. De l'éclatement du graphisme des partitions au $\mathrm{XX}^{\mathrm{e}}$ siècle à l'examen de la valeur plastique des sons, Marie-Aimée Lebreton questionne la notion même d'image sonore qui convoque, par delà l'image mentale ou la représentation graphique des sons, des propriétés géométriques, des territoires sonores, durées plastiques et espace-son.

Un autre type de territoires, où l'on entend le grondement sourd des paysages entropiques de Robert Smithson, se dessine dans l'article de Jacques Leenhardt «Sur l'entropie et le paysage : à propos de Robert Smithson ». Des territoires à l'écart du devenir commercial de l'art s'affirment au cœur de la destruction, sur le fond historique désastreux et désordonné de l'après-guerre, mais aussi dans un mouvement libératoire de sortie du cube blanc, pour creuser, à même le sol, des gouffres où collapsent les significations ordinaires des images et des textes. Il s'agit de retracer un processus où traces, documents et images constituent le champ narratif d'un paysage où la temporalité de l'expérience individuelle s'ouvre au temps cosmique.

Les réajustements de distance focale entraînés par le tournant iconique n'ont pas laissé indemnes les disciplines traditionnellement vouées à l'art : l'esthétique et l'histoire de l'art. Les identités disciplinaires, de plus en plus perméables aux influences venues de l'extérieur, semblent être progressivement prises dans un jeu de transpositions, de reflets et miroitements d'un domaine à l'autre, d'assimilations plus ou moins faciles renvoyant aux oubliettes les différences qui forment la physionomie propre à chaque discipline. Dans « Anti-mémoires : noms, reflets et écritures » de Filippo Fimiani, l'art mémorial public est pensé, en compagnie d'Arthur Danto, en termes de mémoire et anti-mémoire, de construction d'une identité collective, sociale, culturelle et politique constamment renégociée, en proie à des refoulements et oublis qui affectent images, textes et disciplines. Entre le Vietnam Veterans Memorial à Washington et le Monument contre le fascisme de Jochen et Esther Shalev-Gerz, le texte et le contexte des pratiques et usages que convoquent les monuments publics permettent de montrer comment leurs régimes discursifs et iconiques sont pris dans un filet de reflets et d'écarts analysés à l'aune de l'esthétique et de l'histoire.

Au-delà de ces questions institutionnelles et disciplinaires, les images n'arrêtent tout de même pas de provoquer de vives réactions. Depuis la condamnation platonicienne dont on ne se défait jamais complètement, ces derniers prennent tantôt la forme de combats : qu'on pense aux attaques menées par les religions monothéistes contre les idoles, à l'iconoclasme byzantin et aux disputes sanglantes concernant le culte des images ou bien, plus récemment et sur le terrain intellectuel français, la forme de querelles aux affrontements autour de l'(im)possible représentation par ou en images 
de l'horreur des camps de concentration. Tantôt les débats revêtent les armures d'une guerre des images, comme celle dont parle Serge Gruzinsky ${ }^{33}$, de "révolte des images $\|^{34}$ ou encore de clashs : on pense notamment à Iconoclash ou iconocrise, terme par lequel Bruno Latour, mêlant grec et anglais, désigne «le soin pris à dénouer les passions furibondes qu'entraîne l'iconoclasme. $»^{35}$ Dans le catalogue imposant et hétérogène qui accompagne l'exposition Iconoclash, Bruno Latour présentait cette entreprise comme une prise de position contre la haine et le fanatisme, comme exposition qui "s'efforce de suspendre la soif de destruction des images", comme expérience menée au croisement de la science, de l'art et de la religion (2002, ZKM, Karlsruhe) ${ }^{36}$. Ce propos à l'ambition vertigineuse articule religion, science et art, et jusqu'aux nouvelles formes d'iconoclasme représentées par les attaques systématiques portées par l'art contemporain à la représentation même. Plus récemment encore, la violence qui dépasse l'image, qui crève l'écran: le court métrage critique de la soumission des femmes dans l'Islam de Théo Van Gogh, qui mène à son assassinat; les controverses autour des caricatures du Prophète: celles, parues dans le quotidien danois Jyllands-Posten le 30 septembre 2005, à l'origine de menaces de mort et de véritable crise, ou encore le dessin de Plantu, qui, le 3 février 2006, puise dans les ressources de la typographie, autrement dit va au cœur de la tradition islamique, comme le note Bruno Latour, pour dessiner à l'aide du tracé scriptural, des mots enchaînés, l'invisible face du prophète, jouant sur la licence du verbe (la phrase écrite à répétition est « Je ne dois pas dessiner Mahomet»). Puis, le tragique 7 janvier 2015. Une césure dans le temps et dans la narration et peut-être dans la représentation même, qui fit basculer les querelles dans une réalité n'ayant plus rien à voir avec l'image. À la place, c'est des corps humains, des vies qui ont été décimées.

Après le tournant iconique place volontiers les enjeux dans une postérité, une postérité, dont il est impossible de taire les accents tragiques, une postérité qui est aussi une ouverture, ou plus précisément, un réajustement, un rééquilibrage. Il est la marque d'un constat: le régime du tournant iconique est désormais désamorti, seul il ne règle plus aussi fortement le point focal des connaissances. Ni l'art ni la culture ne gagnent à être ramenées aussi fortement au régime visuel. Les enjeux passionnels autour des images, tout le sérieux qu'on leur accorde et qui va jusqu'à se confondre dans la violence aveugle, ne tiennent plus le pari cognitif. En adoptant une perspective ouverte, ce volume issu d'un colloque qui s'est tenu bien avant le 7 janvier 2015, acquiert sans doute aujourd'hui un nouvel éclairage historique et artistique ${ }^{37}$.

\section{NOTES}

1. Ernst Cassirer, "Langage et Art II", in Écrits sur l'art, Euvres, t. 12, Paris, Cerf, 1995, (« Language and Art II », 1942).

2. Paris, Seuil, 1990. Voir aussi Richard Rorty, The Linguistic Turn. Recent Essays in Philosophical Method, Chicago, 1967.

3. "Iconic Turn et réflexion sociétale", Trivium, I, Iconic Turn, 2008, § 2: https:// trivium.revues.org/308\#ftn3. Consulté le 15/12/2016. 
4. Gottfried Boehm, Was ist ein Bild?, Munich, Fink Verlag, 1994.

5. Pour reprendre la proposition de Bernd Stiegler, «Iconic Turn et réflexion sociétale », op. cit., $\S 2$.

6. Gottfried Boehm, W.J.T. Mitchell, « Pictorial versus Iconic Turn. Two Letters », Culture, Theory \& Critic, 2009, 50 (2-3), p. 104.

7. Ibid., p. 105.

8. Ces thèses sont énoncées dans l'article «Par delà le langage ? Remarques sur la logique des images. » («Jenseits der Sprache? Anmerkungen zur Logik der Bilder », in Christa Maar, Hubert Burda (éd.), Iconic Turn. Die Neue Macht der Bilder, Cologne, Dumont, 2004), in Trivium, 1, Iconic Turn, op. cit..

9. Ibid., p. 114.

10. W.J.T. Mitchell, Que veulent les images, Dijon, Les presses du réel, 2014 (What do Pictures Want? The Lives and Loves of Images, University of Chicago Press, 2005).

11. «Pictorial versus Iconic Turn : two letters », op. cit., p. 113.

12. Voir par ex. Hans Belting, Pour une Anthropologie des images, Paris, Gallimard, 2004 (BildAnthropologie. Entwürfe für eine Bildwissenschaft, Munich, Wilhelm Fink Verlag, 2001).

13. Voir Ästhetik und allgemeine Kunstwissenschaft, Stuttgart 1906. En 1906, Dessoir fonde l'influente Zeitschrift für Ästhetik und allgemeine Kunstwissenschaft, qui existe encore aujourd'hui, et dès 1908 la Vereinigung für ästhetische Forschung, société savante, à l'origine des premiers Congrès internationaux d'esthétique.

14. Jean-Paul Sartre, L'imaginaire, Paris, PUF, 1981, p. 162.

15. Henri Lefebvre, Fondements d'une sociologie de la quotidienneté, vol. II, Paris, 1961, p. 290.

16. Philippe Dubois, L'acte photographique et autres essais, Paris, Nathan, 1990, p. 13.

17. L'art et ses agents. Une théorie anthropologique, Dijon, Les presses du réel, 2009 (Art and Agency. An Anthropological Theory, Oxford, Clarendon Press, 1998).

18. Paris, Gérard Monford, 1998. (The Power of Images: Studies in the History and Theory of Response, Chicago, University of Chicago Press, 1989).

19. «Par delà le langage ? Remarques sur la logique des images. ", in Trivium, 1, Iconic Turn, op. cit., §3.

20. Christa Maar, Hubert Burda (éd.), Cologne, Dumont, 2004.

21. Emmanuel Alloa (éd.), Dijon, Les presses du réel 2010 et 2015.

22. Kathrin Busch, Iris Därmann (éd.), Munich, Wilhelm Fink Verlag, 2011.

23. N. Bryson, M. A. Holly, K. Moxey (éd.), New York, Harper Collins, 1991.

24. Jessica Evans, Stuart Hill, Sage Publications, New Delhi, 1999.

25. Petra Maria Meyer (Hrsg.), Acoustic Turn, Wilhelm Fink Verlag, 2010, Don Ihde, Acoustic Technics, Lexington Books, 2015.

26. On pense au développement de projets liés à l'Urban Sound, aux sons de la ville, à la réflexion sur l'écologie sonore et jusqu'à la légitimité récemment accordée au son au théâtre par exemple, dans le cadre du projet Le son du théâtre (CNRS, ENS, Paris 3, UdeM). Voir notamment Raymond Murray Schafer, Le paysage sonore, Wildproject éditions, 2010 [1977] ; Michael Bull, Sounding Out the City: Personal Stereos and the Management of Everyday Life. Oxford. Berg, 2000 ; Frances Dyson, Sounding New Media: Immersion and Embodiment in Arts and Culture. Berkeley: University of California Press, 2009; Steve Goodman, Sonic Warfare. Sound, Affect, and the Ecology of Fear. Cambridge: MIT, 2010 ; Jonathan Sterne, The Audible Past. Cultural Origins of Sound Reproduction, Durham, Duke University Press., 2003.

27. Pour un développement éclairant sur les rapports tournant linguistic-tournant iconique, nous renvoyons vers le riche dossier franceo-allemand déjà évoqué « Iconic Turn », in Trivium, 1 : https://trivium.revues.org 
28. Emmanuel Alloa (éd.), Bildtheorie aus Frankreich. Eine Anthologie, Munich, Wilhelm Fink Verlag, 2011, p. 33. Voir aussi Bildtheorien aus Frankreich. Ein Handbuch, Kathrin Busch, Iris Därmann (éd.), Munich, Wilhelm Fink Verlag, 2011.

29. Karim Ressouni-Demignieux, Dominique Donadieu, Giuseppe Di Liberti, Maddalena Parise, Giuliana Ravviso, Itay Sapir et moi-même.

30. Jacques Morizot, Interfaces: textes et images. Pour prendre du recul de la sémiotique, Presses Universitaires de Rennes, 2004.

31. Muriel Van Vliet, «Bildtheorien aus Frankreich, circonvolutions de la théorie française de l'image ", Regards croisés, $\mathrm{n}^{\circ} 1, \quad 2013$, http://hicsa.univ-paris1.fr/documents/file/ Regards\%20Croises_1.pdf. Consulté le 15/12/2016.

32. Voir Elisa Brilli, «L'essor des images et l'éclipse du littéraire. Notes sur l'histoire et sur les pratiques de l'histoire des représentations ", in E. Brilli, P.-O. Dittmar et B. Dufal (éd.), Faire l'anthropologie historique du Moyen Âge, L'Atelier du Centre de Recherche Historique, n 6, 2010, https:// acrh.revues.org/2028. Consulté le 15/12/2016.

33. Serge Gruzinski, De Christophe Colomb à « Blade Runner », Paris, Fayard, 1990.

34. Gerhard Paul, Aufstand der Bilder. Die NS Propaganda vor 1933, Bonn, Dietz, 1992.

35. Bruno Latour, "Flot et défaut des images : de l'iconoclasme à l'iconoclash ", in Dictionnaire des images, dir. Laurent Gervereau, éd. Nouveau Monde, p. 122.

36. Iconoclash. Beyond the Image Wars in Science, Religion, and Art, catalogue de l'exposition, B. Latour et P. Weibel (éd.), Karlsruhe, ZKM - Cambridge et Londres, MIT Press, 2002.

37. Que soit ici remerciés Elisa Brilli, Thomas Golsenne et Vasso Zachari pour leurs relectures attentives et bienveillantes.

\section{AUTEUR}

TANIA VLADOVA

ESADHAR/EHESS 